\title{
Molecular dynamics simulations reveal the importance of conformational changes, glycosylation, mutations, and drug repurposing for the Sars-Cov-2 spike protein
}

Ludovico Pipitò, Roxana-Maria Rujan, Christopher A. Reynolds, and Giuseppe Deganutti*

Centre for Sport, Exercise and Life Sciences (CSELS), Faculty of Health and Life Sciences, Coventry University, Coventry CV1 2DS, UK

*Corresponding Author ad5288@coventry.ac.uk

\begin{abstract}
The protein responsible for the first steps of SARS-CoV-2 cell invasion, the spike protein, has received much attention in light of its central role during infection. Computational approaches are among the tools employed by the scientific community in the enormous effort to study this new threat. Molecular dynamics (MD) in particular, has been used to characterize the function of the spike protein at the atomic level and unveil its structural features from a dynamic perspective. Here, we review the main findings of MD studies on the spike protein, including flexibility of the stalk region, the role of the glycans on the surface of the S protein, the effect of mutations on biding to ACE2, the change from the down conformation to the up conformation, and progress in drug repurposing.
\end{abstract}

\section{Keywords}

Spike protein; SARS-CoV-2; Molecular dynamics, drug repurposing

\section{Subtitle}

MD simulations of the Sars-Cov-2 spike protein

\section{Introduction}

The year 2019 signalled the start of the worldwide outbreak of Coronavirus Disease (Covid19) from the coronaviridae SARS-CoV-2 virus, which counts around 360 million cases around 
the world with more than 5.6 million certified deaths (WHO dashboard, 27 January 2022). Coronaviridae is an enveloped positive-stranded, non-segmented RNA virus with a genome of about $30 \mathrm{~Kb}$.[1] Coronaviridae are responsible for cardiovascular, hepatic, respiratory, gastrointestinal and neurological diseases, with major symptoms associated with a hyperbolic expression of proinflammatory signals and cytokines such as interleukins, interferon-gamma (IFN- $\gamma$ ), interferon-gamma induced protein 10 (IP-10), macrophage inflammatory protein 1A and 1B (MIP-1A, MIP1-B), platelet-derived growth factor (PDGF), tumour necrosis factor (TNF- $\alpha$ ), and vascular endothelial growth factor (VEGF).[2]

The SARS-CoV-2 infection mechanism depends on the transmembrane spike protein (S protein, Figure 1a,d),[3, 4] a highly conserved structure amongst the coronaviridae family responsible for extracellular binding and cell membrane fusion.[5] It characterizes the shape of this family of viruses, giving it the "solar" crown aspect[4] they are named after. The SARSCoV-2 strain shows a selective affinity for the angiotensin-converting enzyme 2 (ACE2, Figure 1a) receptor, a type 1 transmembrane protein with an external peptidase domain normally responsible for the conversion of angiotensin hormone into angiotensin II.[6]

The $\mathrm{S}$ protein has aroused the interest of medical and pharmaceutical research, to prevent infection and reduce the burden of clinical intervention. It is a homotrimer class I fusion protein, with each protomer composed of domain S1 and S2 (in the prefusion conformation, Figure 1a).[7] The S1 structure is responsible for binding ACE2 (Figure 1a-c), before the conformational change in the stalk-like structure (Figure 1a,d) of the S2 subunit[8] and the subsequent membrane fusion after the cleavage of S1 from S2 by the host transmembrane protease serine 2 (TMPRSS2).[4] The cleavage of the inter-region domain S1/S2 (Figure 1a,d) allows for S2 structural conformation changes necessary for membrane fusion and post-fusion structure adaptation.[5] In the S1 ectodomain (Figure 1d), the apical portion of the S protein, composed of the receptor-binding domain (RBD), the N-terminal domains (NTDs) and two Cterminal domains (CTDs), folds in a hairpin motif that protects the prefusion conformation of S2 from the external environment.[9] A distinguishing feature of the SARS-CoV-2 strain is an insertion in the protease S1/S2 cleavage site region, rich in arginine, which configures a furin recognition site, commonly found in highly virulent influenza viruses.[10]

Several S protein structures have been determined through cryo-electron microscopy (cryo-EM) and X-ray crystallography (Figure 2). These include the inner S1 and the external S2 domains, and indicate two different states in the RBD domain, named "up" and "down", the former determining an active state favourable to ACE2 binding (Figure 1a-c). For S1 to bind ACE2, the RBD must undergo a conformational hinge movement, exposing the hydrophobic 
region in an "up" conformation state.[11, 12] The coronaviridae family has a distinctive morphology characterized by a spherical virion with a diameter of $91 \pm 11 \mathrm{~nm}$ measured at the membrane, on whose surface there are $24 \pm 9 \mathrm{~S}$ trimers unevenly distributed with a prevalence of $97 \%$ of trimers in "down" conformation[13] at room temperature. The RBD is responsible for ACE2-specific binding through an ensemble of 16 well-conserved residues directly interacting with the receptor[14] (Figure 1a-c). Three different sites (Figure 1c), named according to which part of ACE2 they bind, can be distinguished. Site 1 (identified by residue Gln498, Thr500, Asn501, Tyr505) and Site 3 (Asn487 and Phe486) binds to the $\alpha 1$ helix C (Gln24 and Thr27), while Site 2 (Arg403, Tyr453, Leu455, Phe456, and Gln493) binds to the centre of the helix (Asp30, Lys31, His34, Asp38) which is slightly bent outwards, exposing polar amino acids for interaction.[15] The RBD is an important target for the prevention or treatment of the SARS-CoV-2 infection.[4, 16] Post-translational N- and O-glycosylation mask the $\mathrm{S}$ protein epitopes from immune system recognition.[17] A recent cryo-EM derived S protein model revealed that 44 out of 66 potential sites are found to be heavily N-glycosylated in the ectodomain region (Figure 1a).[7]
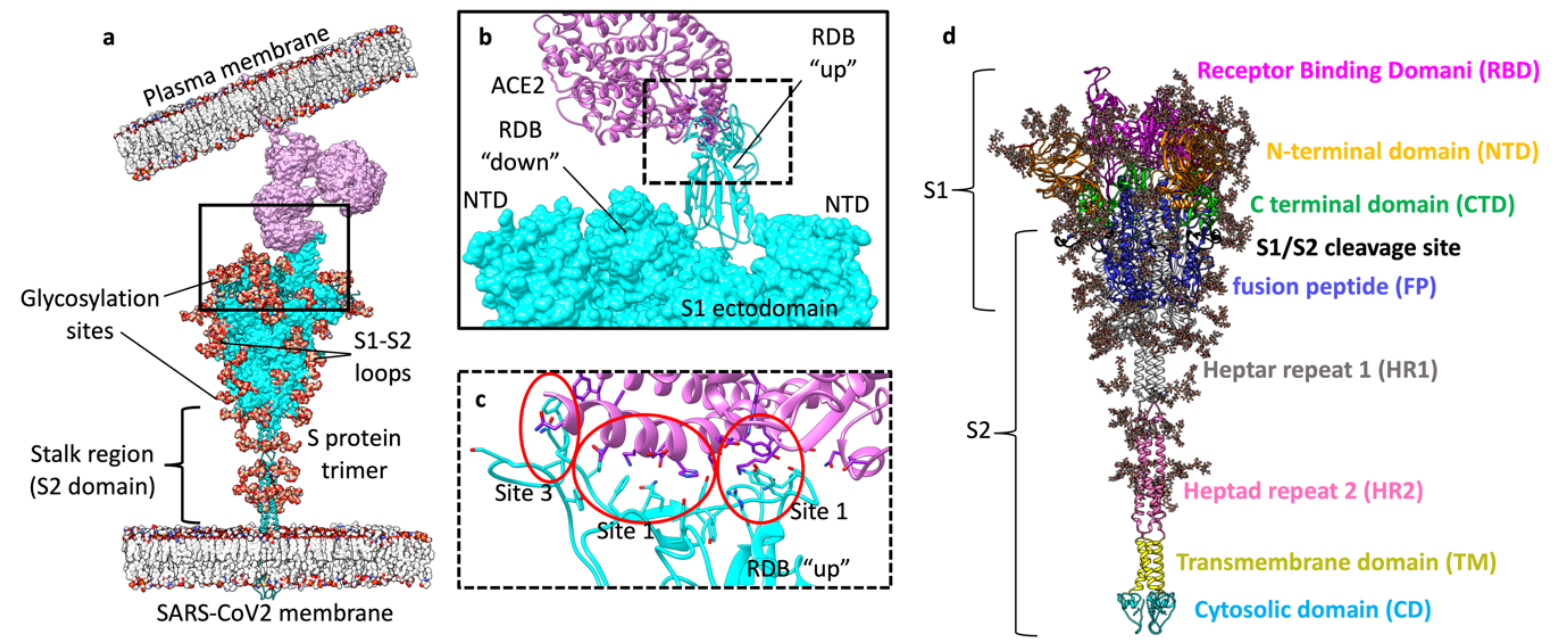

Figure 1. The $S$ protein is the first promoter of SARS-CoV-2 internalization. a) Overall architecture of the complex between $\mathrm{S}$ protein (prefusion conformation, cyan) and ACE2 (violet); glycans on S protein are in van der Waals spheres; the relative positions of the plasma and viral membranes are reported. b) Magnification of the S1 ectodomain (glycans removed for clarity); the RBD in the up conformation is responsible for binding ACE2. c) Magnification of the interface between the RBD and one of the ACE2 monomers; the interactions can be divided according to the relative position into Sites 1 to 3 (red circles). d) Fully glycosylated S protein (https://charmm-gui.org/?doc=archive\&lib=covid19) with the S1 and S2 units 
highlighted; B) The N-terminal domain (NTD), receptor binding domain (RBD), C-terminal domain (CTD), S1/S2 cleavage site, fusion peptide (FP), heptad repeat 1 (HR1), heptad repeat 2 (HR2), transmembrane Domain (TM ), cytosolic domain (CD) are reported. Glycans are shown in licorice.
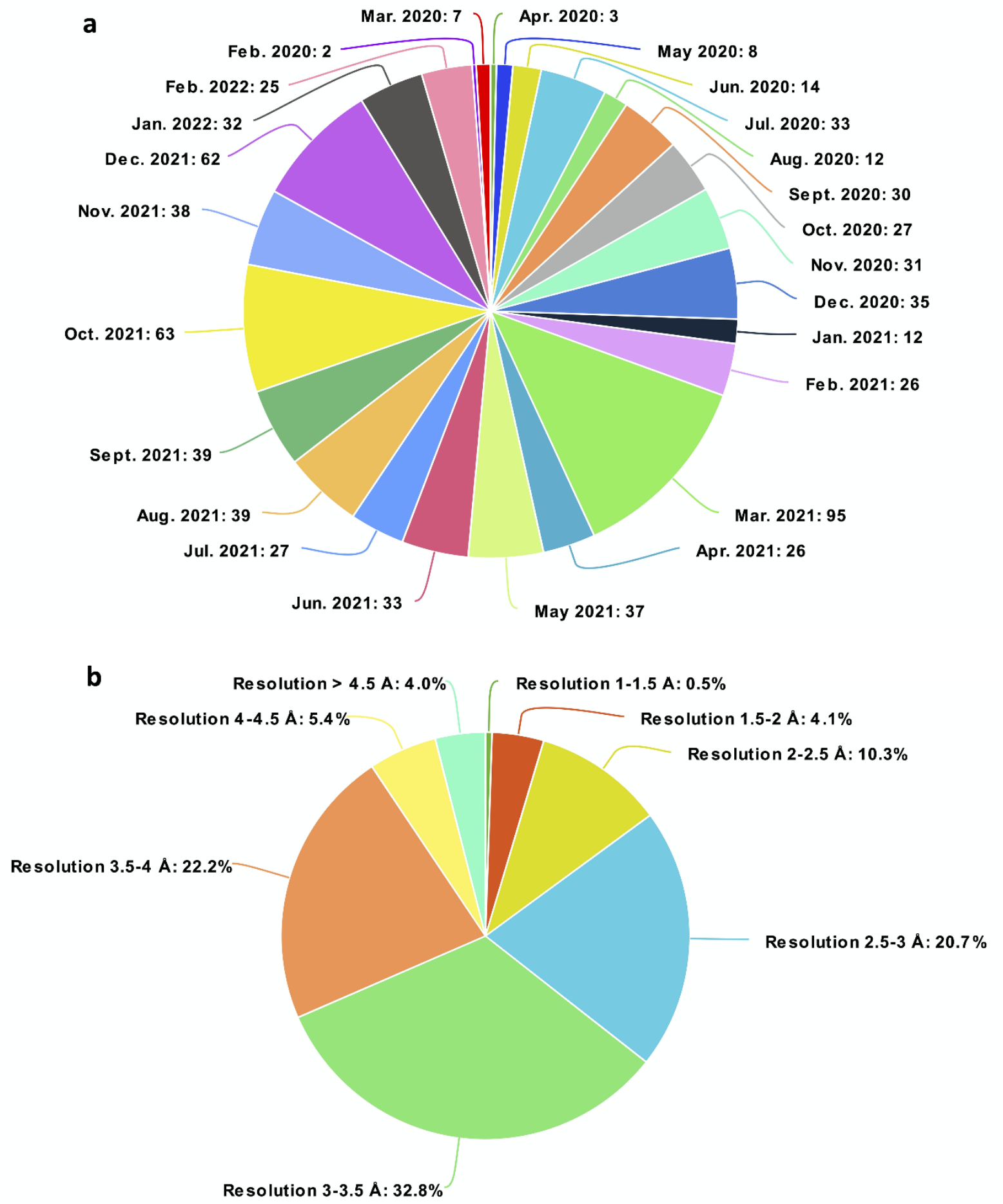
Figure 2. S protein structures deposited in the protein data bank. a) Spike protein structures released and ordered according to month and year of publication from the Protein Data Bank;[18] b) S protein structures only, ordered according to resolution.

Molecular dynamics (MD) is a computational technique that simulates the evolution over time of (bio)molecular structures. It represents a state-of-the-art tool for biophysical studies and structure-based drug design[15] as it describes the positional changes of the atoms of a chemical system that explicitly includes water, ions and other biological components such as protein, membrane, nucleic acids,[19] allowing the conformational exploration of biological structures. The evolution over time is simulated by integrating Newton's classical equation of motion for each atom of the system. The result of this many-particle motion is a trajectory, from which it is possible to extrapolate thermodynamic, kinetics, and physical properties through statistical mechanics.[20] One of the advantages of MD is to overcome the unnatural rigidity that characterizes X-ray crystallography and cryo-EM structures, allowing the investigation of possible cryptic (hidden) binding pockets, allosteric effects, and structural changes in response to binding.

In this review, we recapitulate the MD studies that have expanded our knowledge of the SARS-CoV-2 S protein flexibility and antibody recognition and summarise their contribution to drug repurposing campaigns.

\section{Molecular dynamics simulations uncover the $S$ protein flexibility}

Since the first cryo-EM structures of the $\mathrm{S}$ protein became available to the scientific community, it has been possible investigate the conformational changes and the dynamic processes involving the $\mathrm{S}$ protein through MD simulations. One of the limits of the S protein structure experimentally determined is the scarcity of structural information about the posttranslational glycosylation, due to the high dependency on the organism used for protein expression. To address this aspect, Woo and his group modelled a set of complete and fully glycosylated (Figure 1) S protein structures[21] in accordance with Wrapp et al.'s suggestion of the S protein glycosylated in 44 out of 66 possible sites in the ectodomain region.[7]

A study by Choi and co-workers,[22] showed that the S1 domain displays structural compactness, while the stalk is characterized by two flexible portions, at the heptad repeat 2 (HR2) linker and the heptad repeat transmembrane portion (HR2-TM) respectively. Such findings are in line with the experimental observation that the $\mathrm{S}$ protein can tilt up to $90^{\circ}$ toward the membrane, with inclination of $48^{\circ}$ (to the membrane normal) being most likely to occur. 
Such movements might favour ACE2 binding.[23] Through the use of nontargeted parallel cascade selection MD (nt-PaCS-MD), the variational approach to Markov processes (VAMP) analysis indicated distinct conformational changes in cleaved SARS-CoV-2-spike models at the level of residues Val705-Asp1146 and residues Ser816 -Asp1146.[24] These changes are necessary to expose the fusion peptide (FP) and rearrange the region between residues Ile818 - Val826 of the FP into an outward facing helical structure which might mechanically initiate membrane fusion.

MD studies of SARS-CoV-2 have indicated accentuated flexibility compared to its predecessor SARS-CoV in segments of the RBD implicated in the molecular recognition of ACE2, more precisely in the region comprising residues Gln474-Gly485, Cys488-Phe490, and Ser494-Tyr505 (Sars-Cov-2 numbering) of the RBD, which enhances binding to the ACE2 receptor.[25] MD simulations were used to appraise the effect of mutations such as Asn501Tyr and Glu484Lys, which improved the binding to ACE2 by -4.5 and $-1.3 \mathrm{kcal} / \mathrm{mol}$ respectively, as determined by free energy perturbation (FEP). The flexibility of RBD in the "up" conformation has been proposed as a determinant for the high propensity of SARS-CoV-2 to reach ACE2, giving rise to the high infectivity associated with SARS-CoV-2.[25] Interactions not observed in the static cryo-EM or X-ray crystal structures but observed in MD simulations involve residues Ile21, Gln24, Thr27, Phe28, Asp30, Glu35, Asp38, Ala80, Met82, and Tyr83 of ACE2.[26] A study by Barros et al.[27] indicated that ACE2 presents great motility when in contact with $\mathrm{S}$ protein. This favours a rotation of the catalytic zinc-binding peptidase domain (PD) along the transmembrane domain axis. This large shift was enhanced by five glycan residues bound to Asn53, Asn90, Asn103, Asn322, and Asn546 of ACE2, with Asn53 involved in both intramolecular homodimer and heterodimer contacts.[27, 28] Williams and coworkers[29] suggested that in the RBD:ACE2 interaction pattern, residues Phe486, Asn487, and Tyr489 are responsible for the adaptive flexibility of RBD in establishing strong interactions with ACE2. At the same time, this study demonstrated how mutations in that RBD sub-region are crucial in the selective pressure of the virus, altering the flexibility of RBD and interfering in intra-monomer interactions within the RBD.[29] From a geometric perspective, effective interaction between SARS-CoV-2-spike and ACE2 would occur at an angle of inclination between the apical portion of RBD "up" and ACE2 of at least 52 . [30] Such MD results indicated that RBD "up" conformations have a large degree of manoeuvre to achieve sufficient residue exposure for ACE2 binding.

An intriguing quantum-mechanics/molecular mechanics (QM/MM) analysis, performed on an MD trajectory that included the residues on the RBD binding motif (RBM) 
and ACE2, proposed the importance of the hydrogen bonds involving Gly502, Lys417, Asn487, Thr500, Gln493, and Tyr505 on S protein RBD in strengthening the molecular recognition necessary for the infectious mechanism.[31] These findings suggested that hydrogen bonding may be considered as the major contributor for selective pressure for SARS$\mathrm{CoV}-2$ and should be taken in consideration as a key aspect for further molecular analysis or drug development.

Furthermore, MD models can describe the effects of mutations on the binding energies between proteins or ligands in a predictive way, anticipating drug design strategies or describing concerning viral variants. A study from Luan et al.[32] estimated the effect of the Asn501Tyr mutation, which engaged the hydrophobic benzene ring on residue Tyr41 on ACE2. Using FEP, Luan's results indicated an increased affinity for the ACE2 receptor due to an increase in the binding affinity. Such mutation on Tyr501 shifts RBD's orientation upward toward ACE2, engaging with both residues Tyr41 and Lys 353 on the receptor, favouring a bound state.

Interestingly, based on observations on the prognosis of smokers, Oliveira et al.[33] investigated the possible engagement between $\mathrm{S}$ protein with nicotinic acetylcholine receptors (nAChRs). The authors hypothesize that molecular recognition between RBD and nAChRs can contribute to the hyperinflammatory effects observed in Covid-19. MD and molecular mechanics Poisson-Boltzmann surface area (MM-PBSA) analysis identified a ProlineArginine-Arginine-Alanine (PRRA) motif on the RBD between residues Try674 to Arg685, capable of recognizing different nAChRs. Oliveira's work suggested that the binding between RBD and nAChRs could exacerbate the inflammatory response, while nicotine could alleviate Covid-19 hyperinflammatory effects.

\section{Molecular dynamics simulations to explore rare $\mathbf{S}$ protein conformational changes}

Large protein conformational rearrangements usually take place in the millisecond or second time scale, far beyond the time simulated in MD, which is usually within tens of microseconds. The implication of this is that rare conformational changes can be missed. It is, therefore, necessary to apply enhanced or adaptive sampling algorithms to overcome this intrinsic limitation of the sampling that can be achieved. From this perspective, weighted ensemble (WE) MD allows sampling rare events,[34] drastically increasing the computational efficiency. With WE, multiple simulations are run in parallel and the trajectories that explored new values of a metric decided a priori (a distance between atoms in the simplest case) are retained and 
replicated, thus minimizing the randomness of conformational exploration. By using the WE path-sampling strategy, Sztain et al.[35] were able to simulate the transition state of RBD from "down" to "up", uncovering the crucial role of several glycan residues in allosterically stabilizing the "up" state. While Asn165 and Asn264 shield the RBD, acting as an "up" state stabilizer, Asn343 pushes the RBD to the final "up" state by interacting with residues Phe490, Tyr489, Phe456, and Arg457 on the interaction portion of the ACE2 binding motif.[35] An approach combining WE and artificial intelligence (AI) was adopted by Casalino et al. [36] to evaluate metastable conformations during the binding between fully glycosylated S protein and ACE2.[36] This confirmed the role that the two N-glycan residues linked to Asn165 and Asn234 have in modulating the dynamics of the S protein's RBD, contributing to the axial mobility of ACE2 while triggering the opening of the RBD in a "hand jive" motion. Yao and co-workers,[37] analysed the molecular architecture of SARS-CoV-2, from cryo-electron tomography (cryo-ET) and subtomogram averaging (STA), highlighting the complex composition of $\mathrm{N}$-glycans, which is the result of unions between branched oligomannose and hybrids units. Such complex glycan ramification also appears to be present on Asn234, whose allosteric role in the conformational change of RBD from "down" to "up" has been demonstrated by Amaro et al.[35]

In a separate study, all-atom steered MD (SMD) forced the RBD from "down" to "up" and highlighted the conformational changes that occur during the breaking of the salt bridges between RBD and the neighbouring protomers with the hydrogen bonds that keep RBD in an inactive "down" state.[38] These intramolecular salt bridges, Lys378-Glu988 and Lys386Asp985 within the S2 domain, and Glu516-Lys202 within the NTD are mainly responsible for the inactive "down" state of the monomers and prevent the interactions with ACE2. Data obtained through targeted molecular dynamics (TMD) have shown how glycans on RBD residues Asn165, Asn234, and Asn343, can act as position locker for the active "up" conformation,[39] stabilizing a set of interdomain salt bridges involving Lys417, Arg408, and Lys378. Furthermore, glycans on Asn165 and Asn234 were proposed as shielding the epitopes (Figure 3), while locking RBD in the "up" state.[40]

A remarkable effort was made by Zimmerman et al.,[41] to explore drastic conformational changes through Markov-state models (MSMs) combined with the computational power provided by "citizen-scientists" from the "Folding@home" project (http://foldingathome.org), Very long time scale simulations, in conjunction with the FAST algorithm, described drastic conformational changes on the S protein which opened the RBD 
from its "down" to the "up" state, while the RBD domain twisted outward, exposing new cryptic epitopes (see below).

\section{Hide and seek: the hunt for epitopes through MD}

Access to the S protein epitope(s) is necessary for antibody binding (Figure 3).[42] From this standpoint, long MD simulations might unveil cryptic epitopes. Sikora et al.[42] performed an extensive simulation of four $\mathrm{S}$ proteins embedded in a membrane, for a total of $2.5 \mu \mathrm{s}$. The resulting trajectories were analysed through simulated illumination analysis and rigid docking of the antibody CR3022. In the illumination analysis, randomly oriented rays emanated from a half-sphere at the centre of mass of the S protein. Rays are then absorbed by the first heavy atom they meet within $1.5 \AA$. Single $\mathrm{S}$ protein structures are collected every $10 \mathrm{~ns}$. To evaluate the shielding effect of glycans, the analysis was replicated without their presence. The results indicated that glycans reduce the $\mathrm{S}$ protein accessibility up to $80 \%$, with the most marked effect occurring in the stalk region close to the viral membrane. An interesting ab initio epitope mapping method was used by Serapian et al.[43] Possible epitopes were classified according to the coupling energy with the rest of the structure, which identifies sites on the $\mathrm{S}$ protein surface which are at lower binding energy levels and could possibly be energetically preferred bound states. This method suggested that residues forming an epitope prefer to form molecular interactions with external elements.[43] The data agrees with the experimentally detected epitope recognized by antibodies CR3022, 4A8, S309, and EY6A.

The use of small molecules or cosolvents as probes in MD simulations (mixMD) increases the chance to discover cryptic niches or epitopes on the surface of a protein. Through MixMD it is possible to map interesting interaction sites by considering the frequency of contacts between protein and probe, resulting in a volumetric map. Using a concentration around 1-5\% cosolvent usually improves the sampling of hot spots for interactions without denaturing the protein. Pyrimidine (Py), acetonitrile, and isopropanol were used to discover possible docking niches on the interface between RBD and ACE2, to inspire the drug design of antagonists or antibodies.[44] Py showed the most relevant volumetric maps within the RBM that spans from residue Gln498 to residue Tyr505. Knowledge of these types of interactions, in conjunction with molecular mechanics with generalised Born surface area solvation (MMGBSA) analysis, lead drug design processes and virtual screening in concordance with experimental data from antibodies, has suggested a set of new molecules (DB02651, DB03714, DB08248 and DB14826) as possible RBD interaction modulators. 


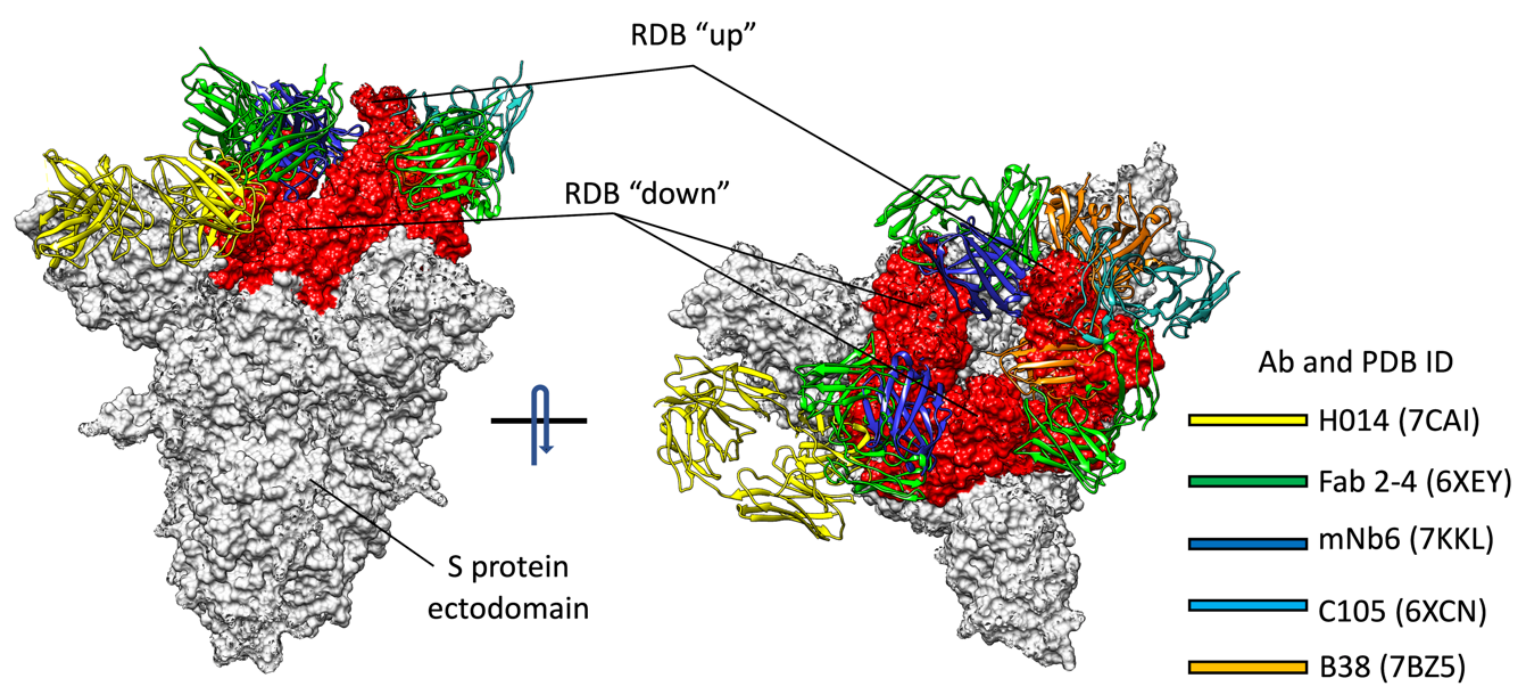

Figure 3. Human antibodies can bind to different $\mathbf{S}$ protein epitopes. The binding position of five human antibodies on the surface of the S protein, coloured according to the legend. Antibody names and protein data bank database IDs are reported in the legend. The S protein is represented in white surface, with the RBD in red.

\section{Computer-aided drug repurposing to tackle COVID19: the role of molecular dynamics} simulations

A possible strategy to overcome barriers in the research and development of new active compounds is through drug repurposing of existing formulations for a different therapeutic indication. Drug repurposing is usually characterized by a substantial variation from its original use. Since the COVID-19 outbreak, the alarming spread of the virus and the gravity of the infection led the scientific and medical community to seek rapid responses. A huge drug repurposing effort was put in place,[45] worldwide, to shorten as much as possible the approval time of therapeutics against SARS-CoV-2 validated targets. Necessarily, the S protein has been one of the most investigated COVID-19 therapeutic targets due to its unique function and central role in the early stage of infection.

The general idea behind targeting the spike protein is to act as a preventive defence against infection, with the intent of minimizing the risk of triggering a potentially dangerous over-reaction of the immune system, reducing de facto the burden on the public health sector. In May 2020, news about the efficacy against COVID-19 of hydroxychloroquine (HCQ) originated in China, and the use of HCQ and azithromycin (ATM) was indicated as a possible front-line treatment. Simulations indicated that HCQ and ATM would have a synergistic effect in the treatment of the infection, where HCQ acts as a competitive binder against gangliosides, 
another proposed receptor for the S protein, and ATM interacts with the tip of SARS-CoV-2spike.[46] Although these results seemed promising, the outcomes of clinical trials appeared highly controversial and the hypothesis of adopting the combined HCQ and ATM therapy has been abandoned.

To face the threat of SARS-CoV-2 and its mutations, including the British (alpha) variant,[47] large companies such as Pfizer BioNTech and AstraZeneca[48, 49] have developed vaccines capable of activating an immunogenic response against the S protein. A global vaccination campaign has started, with more vaccines currently under development all around the world.[50] However, as low-income countries struggle to have access to vaccines and immunosuppressed and allergic subjects cannot take advantage of the protection offered, alternative therapeutic approaches are still needed. Also, despite the high efficacy of vaccines, the full compliance of the population of high income countries is yet to be reached, due to the limited knowledge of the long-term effects of new mRNA technologies and their implementation. $[49,51]$ In this scenario, drug repositioning could bring many advantages in terms of risk control and unwanted side effect management - because repurposed drugs have already passed safety assessments. Understandably, antiviral agents were among the first agents to be tested against COVID-19. This approach led to the approval of remdesivir as the first treatment for hospitalized patients, $[52,53]$ but not without controversies, due to uncertain outcomes of many clinical trials.[54]

The general approach to drug repurposing ideally starts with three steps: identifying the candidate molecule to generate the hypothesis, preclinical studies of the candidate molecule, and evaluation of efficacy in phase II clinical trials.[55] A wide set of computational techniques and software, which falls under the category of computer-aided drug design (CADD), is routinely used during the first step of drug repurposing to pinpoint potential drug candidates. CADD includes a plethora of ligand-based and structure-based approaches, involving target structural validation, binding energy evaluation in both static and dynamic models, and pharmacokinetics prediction. Long MD simulations have become a state-of-art computational tool in CADD[15] as they represent the best tool to validate in silico results of molecular docking and virtual screening campaigns. Here we report insights from MD simulations applied to the discovery of potential drugs able to interfere with the binding between RBD and ACE2. Only molecules tested both in vitro and in silico are reported.

One of the first computational work on SARS-CoV-2 proposed denopamine (Table 1A), bometolol, and rotigaptide as possible inhibitors of S protein-ACE2 binding.[56] The authors tested denopamine in vitro, observing a diminishing of RBD binding at denopamine 
concentrations $>100 \mu \mathrm{M}$.[57] An in silico study highlighted simeprevir and lumacaftor as putative RBD binders.[58] Lumacaftor (Table 1B) was subsequently proved to weakly bind to S protein with an $\mathrm{IC}_{50}$ of $84 \pm 4 \mu \mathrm{M}$, although showing a good inhibition profile in Vero-E6 assays.[59] Simeprevir (Table 1C) reduces the cellular viral load, synergizing with Remdesivir, but this effect was attributed to a direct action on the main protease and the RNA-dependent RNA polymerase (RdRp).[60] Post-docking MD simulations identified KT203, BMS195614, KT185, RS504393, and GSK1838705A (Table 1G-H), five compounds from the SigmaAldrich library of pharmacologically active compounds (LOPAC), as potential binders of the S protein.[61] A retrospective MD investigation on arbidol (Table 1I), a therapeutic agent approved in China and Russia for influenza, showed an inhibitor effect on the original SARS spike protein;[62] they proposed arbidol intercalated between different spike protein subunits, and so affecting the trimerization of the $\mathrm{S}$ protein.[63] Docking and MD simulations performed by ourselves[19] and others[64] proposed Nilotinib (Table 1J) as a potential binder of the RBD or disruptor of the RBD-ACE2 complex. The anti-SARS-CoV potential of nilotinib was first reported in 2016 in the early stages of infection by inhibiting viral fusion at the endosomal level.[65] A couple of years later further results pointed out an action of nilotinib and other Abl kinase inhibitors, on the virus-cell membrane fusion.[66] In a recent study, the $\mathrm{EC}_{50}$ of imatinib was quantified as $1.44 \mu \mathrm{M}$ and $3.06 \mu \mathrm{M}$ in Vero-E6 cells and human respiratory cells respectively.[67] Therefore, no experimental evidence for imatinib binding to RBD has been reported. The same goes for nafamostat, which we suggested as a putative RBD binder,[19] but it is proposed to act as a transmembrane serine protease 2 (TMPRSS2) inhibitor in the lownanomolar range.[68, 69]

Table 1. Summary of the drugs, recently identified as protective against SARS-CoV-2 in vitro, proposed as RBD binders by MD simulations.

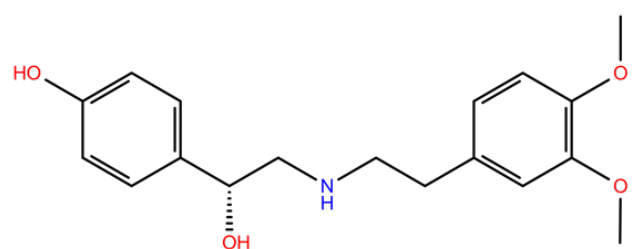

Denopamine: cardiotonic drug acting as an agonist at $\beta 1$ adrenergic receptor; used in the treatment of angina[70]

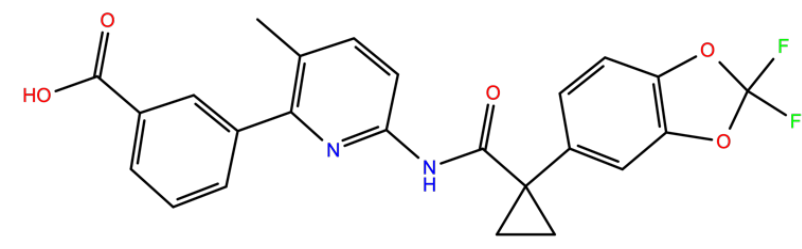

Lumacaftor: used for the treatment of cystic fibrosis in patients that present the F508del in the CFTR (cystic fibrosis transmembrane conductance regulator) proteins;[71] $\mathrm{IC}_{50}$ of $84 \pm 4 \mu \mathrm{M}$ towards the $\mathrm{S}$ protein 


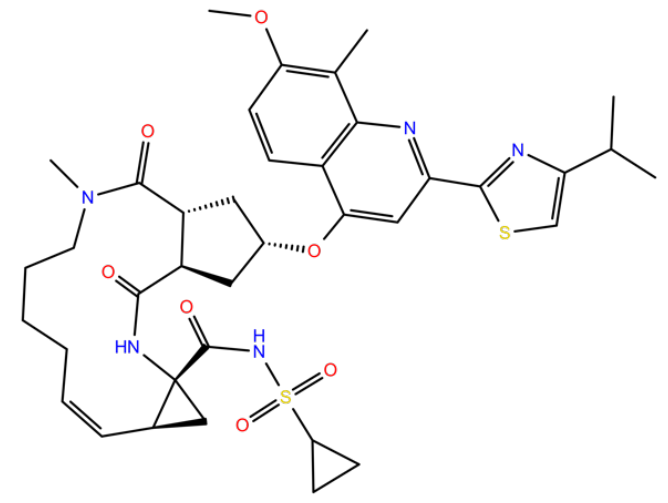

Simeprevir: inhibitor of the hepatitis $\mathrm{C}$ virus (HCV) NS3/NS4A protease [72]

$\mathrm{IC}_{50}$ of $9.6 \pm 2.3 \mu \mathrm{M}$ towards the $\mathrm{M}^{\text {pro }}$ and an $\mathrm{IC}_{50}$ value of $5.5 \pm 0.2 \mu \mathrm{M}$ towards the $\mathrm{RdRp}$ (RNA-dependent RNA polymerase)[60]

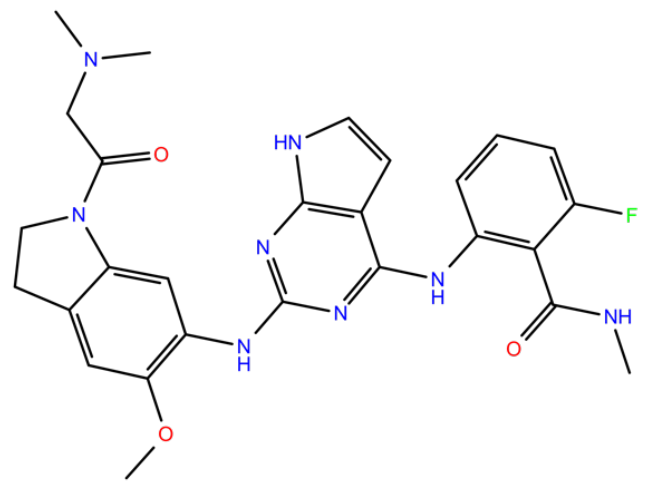

GSK1838705A: inhibitor of the insulin-like growth factor1 receptor (IGF-IR), insulin receptor and anaplastic lymphoma kinase (ALK)[73]<smiles>CC1(C)CC=C(c2cnc3ccccc3c2)c2cc(C(=O)Nc3ccc(C(=O)O)cc3)ccc21</smiles>

BMS195614: antagonist of the retinoic acid receptor (RAR) [74]

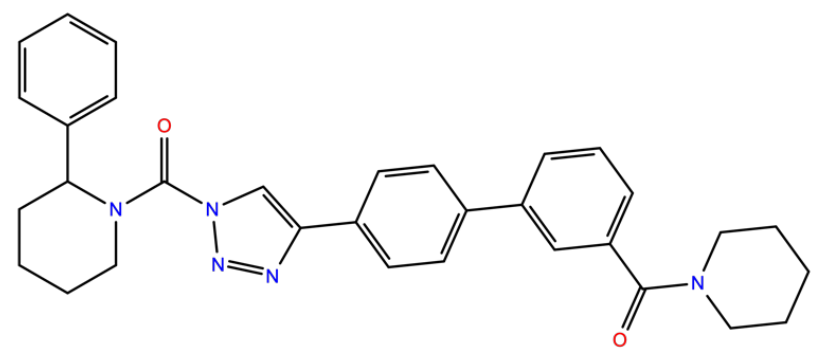

KT185: inhibitor of $\alpha / \beta$-hydrolase domain-containing 6 (ABHD6) in the brain and liver of mice [75]<smiles>Cc1ccc2c(c1)C1(CCN(CCc3nc(-c4ccccc4)oc3C)CC1)OC(=O)N2</smiles>

RS504393: antagonist of the CC2 chemokine receptor [76]

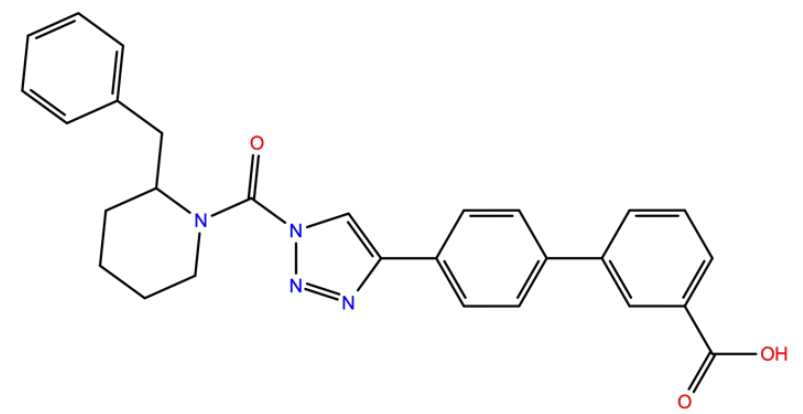

KT203: inhibitor of ABHD6 activity in the liver of mice 
I.

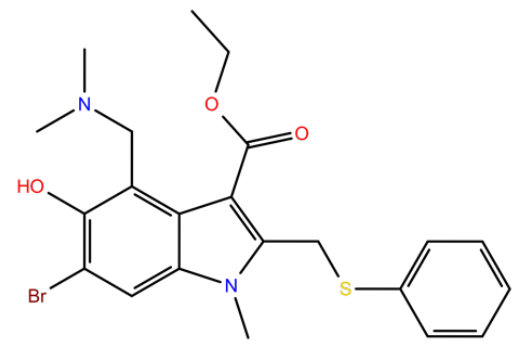

Arbidol: used as treatment for influenza and other respiratory infections in Russia and China [63]
$\mathrm{J}$.<smiles>Cc1cn(-c2cc(NC(=O)c3ccc(C)c(Nc4nccc(-c5cccnc5)n4)c3)cc(C(F)(F)F)c2)cn1</smiles>

Nilotinib: a BCR-ABL tyrosine kinase inhibitor used for the treatment of chronic myelogenous leukemia (CML)[77]

\section{Perspectives for Targeting the Spike Protein}

Although the worldwide effort to discover approved drugs to repurpose against the SARSCoV-2 S protein, to date no MD-based study has delivered working hypotheses resulted in clinical trials. Open access COVID-19 drug repurposing databases[78, 79] are a precious source of information but do not consider potential therapeutic agents proposed in silico, thus, there is a coordination gap between theoretical and experimental scientific communities.[80] From a technical perspective, the amount of MD sampling to confirm molecular docking predictions has been generally limited to the time scale of few tens of nanoseconds and this has probably produced numerous in silico false positives, undermining the credibility of computation studies. Simulations over tens or few hundreds of nanoseconds showing a docking complex as stable should not be faithfully trusted. For example, a molecule with a residence time of few microseconds (way longer than usual MD post docking simulations) and an optimistic binding $\mathrm{k}_{\text {on }}$ of $\approx 10^{7} \mathrm{M}^{-1} \mathrm{~s}^{-1}$ would have a kinetic affinity of about $10^{-2} \mathrm{M}$ and therefore would not be a binder despite the indication provided by MD.

The discrepancy between the time scale of the real world and the simulated models can be partially overcome with end-state methods such as the MM-PBSA or MM-GBSA,[81] which can quantify the binding free energy using short MD simulations. However, the accuracy of these methods is system dependent and usually best suited for comparisons between congeneric ligands[82] rather than very different chemotypes, as is usually required by repurposing strategies. Enhanced MD sampling techniques such as metadynamics[83] speed up the time required to dissociate docking complexes and allow estimation of the stability of the bound ligand; these techniques can therefore aid in recognising docking false positives. 
We screened in silico more than 2000 approved small molecules.[19] After the docking and post docking MD simulations of the best-ranked compounds docked in situ to the RBD, we performed a further step consisting of simulating the encounter of the RBD and ACE2 in the presence of the best compound observed, cefsulodin, bound to RDB. Despite the stability displayed during the cefsulodin / RBD simulations, ACE2 easily displaced the proposed ligand in half of the supervised MD (SuMD $[84,85])$ replicas. Increasing the complexity of the modelled system highlighted the limit of common computational protocols for correctly selecting small molecules able to hinder the recognition between $\mathrm{S}$ protein and ACE2. Therefore, dynamic approaches that consider the formation of the ternary systems between $\mathrm{S}$ protein, ACE2 and the potential binding inhibitor should be routinely considered. Besides this, structure-based drug repurposing strategies should take into account the fully glycosylated S protein and the discovery of allosteric sites on the RBD to overcome the possible undruggability of the binding surface for ACE2. The extreme flexibility of the glycans on the surface of the S protein and the effective steric hindrance they offer affect the ability of antibodies or potential therapeutic molecules to bind to a sufficiently exposed epitope.[86] To combat this, Haji-Ghassemi et al. suggested searching for drugs to target this shield[87] this is a different approach from the traditional protein-oriented one.

\section{Conclusion}

As a state-of-the-art computational technique, MD has been employed to interrogate the structure and function of the S protein at the atomic level to understand how the S protein's inherent flexibility modulates the binding to ACE2 receptors and, therefore, SARS-CoV-2 virulency. MD suggested unexpected flexibility in the stalk region of S2, the role of glycans on the S protein surface, and the contribution of single residues on the RBD to the interactions with ACE2. MD indicated important dynamics and structural elements such as the minimum angulation required for molecular recognition between ACE2 and RBD, the effects of mutations on the binding capacity of the $\mathrm{S}$ protein, as well as the structural and protective role of glycans. Through MD it was possible to understand the motions that open RBD from the "down" to "up" conformation before ACE2 recognition, revealing numerous cryptic pockets that are possible targets of new drugs.

As a future perspective, we believe there is scope for an increasingly important contribution of MD in the study of Antibodies and their rational development as therapeutic agents. Also, MD contributed to rationalizing in vitro data on potential $\mathrm{S}$ protein binding antagonists, but with limited utility in drug repurposing. Approaches to address COVID-19 
start to fade away from drug repurposing and the $\mathrm{S}$ protein to more classic rational strategies to target functional viral proteins, as demonstrated by the main protease (Mpro) inhibitor nirmatrelvir, the first oral anti-COVID-19 drug approved by FDA. In this scenario, it is plausible that MD will regain a central role in aiding the development of future new classes of therapeutics against SARS-CoV-2. 
Bibliography

1. Siddell, SG. . (1995). The coronaviridae: an introduction. In Siddell SG. ed; The Coronaviridae. Springer US. 1-10.

2. Ragab, D., Salah Eldin, H., Taeimah, M., Khattab, R., Salem, R. (2020). The COVID-19 Cytokine Storm; What We Know So Far. Front. Immunol., 11, 1446.

3. Bosch, BJ., van der Zee, R., de Haan, CAM., Rottier, PJM. (2003). The coronavirus spike protein is a class I virus fusion protein: structural and functional characterization of the fusion core complex. J. Virol., 77, 8801-11.

4. Al Adem, K., Shanti, A., Stefanini, C., Lee, S. (2020). Inhibition of SARS-CoV-2 Entry into Host Cells Using Small Molecules. Pharmaceuticals (Basel), 13

5. Fan, X., Cao, D., Kong, L., Zhang, X. (2020). Cryo-EM analysis of the post-fusion structure of the SARS-CoV spike glycoprotein. Nat. Commun., 11, 3618.

6. Tikellis, C., Thomas, MC. (2012). Angiotensin-Converting Enzyme 2 (ACE2) Is a Key Modulator of the Renin Angiotensin System in Health and Disease. Int. J. Pept., 2012, 256294.

7. Wrapp, D., Wang, N., Corbett, KS., Goldsmith, JA., Hsieh, C-L., Abiona, O., ... McLellan, JS. (2020). Cryo-EM structure of the 2019-nCoV spike in the prefusion conformation. Science, 367, 1260-3.

8. Lan, J., Ge, J., Yu, J., Shan, S., Zhou, H., Fan, S., ... Wang, X. (2020). Structure of the SARS-CoV-2 spike receptor-binding domain bound to the ACE2 receptor. Nature, 581, 215-20.

9. Benton, DJ., Wrobel, AG., Xu, P., Roustan, C., Martin, SR., Rosenthal, PB., ... Gamblin, SJ. (2020). Receptor binding and priming of the spike protein of SARS-CoV2 for membrane fusion. Nature, 588, 327-30.

10. Shang, J., Wan, Y., Luo, C., Ye, G., Geng, Q., Auerbach, A., Li, F. (2020). Cell entry mechanisms of SARS-CoV-2. Proc. Natl. Acad. Sci. USA, 117, 11727-34.

11. Cai, Y., Zhang, J., Xiao, T., Peng, H., Sterling, SM., Walsh, RM., ... Chen, B. (2020). Distinct conformational states of SARS-CoV-2 spike protein. Science, 369, 1586-92.

12. Henderson, R., Edwards, RJ., Mansouri, K., Janowska, K., Stalls, V., Gobeil, SMC., ... Acharya, P. (2020). Controlling the SARS-CoV-2 spike glycoprotein conformation. Nat. Struct. Mol. Biol., 27, 925-33.

13. Ke, Z., Oton, J., Qu, K., Cortese, M., Zila, V., McKeane, L., ... Briggs, JAG. (2020). Structures and distributions of SARS-CoV-2 spike proteins on intact virions. Nature, 588, 498-502.

14. Chen, Y., Guo, Y., Pan, Y., Zhao, ZJ. (2020). Structure analysis of the receptor binding of 2019-nCoV. Biochem. Biophys. Res. Commun.,

15. Deganutti, G., Moro, S., Reynolds, CA. (2020). A Supervised Molecular Dynamics Approach to Unbiased Ligand-Protein Unbinding. J. Chem. Inf. Model., 60, 1804-17.

16. Chi, X., Yan, R., Zhang, J., Zhang, G., Zhang, Y., Hao, M., ... Chen, W. (2020). A neutralizing human antibody binds to the N-terminal domain of the Spike protein of SARS-CoV-2. Science, 369, 650-5.

17. Walls, AC., Tortorici, MA., Frenz, B., Snijder, J., Li, W., Rey, FA., ... Veesler, D. (2016). Glycan shield and epitope masking of a coronavirus spike protein observed by cryo-electron microscopy. Nat. Struct. Mol. Biol., 23, 899-905.

18. Berman, HM., Westbrook, J., Feng, Z., Gilliland, G., Bhat, TN., Weissig, H., ... Bourne, PE. (2000). The protein data bank. Nucleic Acids Res., 28, 235-42. 
19. Deganutti, G., Prischi, F., Reynolds, CA. (2021). Supervised molecular dynamics for exploring the druggability of the SARS-CoV-2 spike protein. J. Comput. Aided Mol. Des., 35, 195-207.

20. Padhi, AK., Rath, SL., Tripathi, T. (2021). Accelerating COVID-19 Research Using Molecular Dynamics Simulation. J. Phys. Chem. B,

21. Woo, H., Park, S-J., Choi, YK., Park, T., Tanveer, M., Cao, Y., ... Im, W. (2020). Developing a Fully Glycosylated Full-Length SARS-CoV-2 Spike Protein Model in a Viral Membrane. J. Phys. Chem. B, 124, 7128-37.

22. Choi, YK., Cao, Y., Frank, M., Woo, H., Park, S-J., Yeom, MS., ... Im, W. (2020). Structure, Dynamics, Receptor Binding, and Antibody Binding of Fully-glycosylated Full-length SARS-CoV-2 Spike Protein in a Viral Membrane. BioRxiv,

23. Choi, YK., Cao, Y., Frank, M., Woo, H., Park, S-J., Yeom, MS., ... Im, W. (2021). Structure, Dynamics, Receptor Binding, and Antibody Binding of the Fully Glycosylated Full-Length SARS-CoV-2 Spike Protein in a Viral Membrane. J. Chem. Theory Comput., 17, 2479-87.

24. Remington, JM., McKay, KT., Ferrell, JB., Schneebeli, ST., Li, J. (2021). Enhanced sampling protocol to elucidate fusion peptide opening of SARS-CoV-2 spike protein. Biophys. J., 120, 2848-58.

25. Pavlova, A., Zhang, Z., Acharya, A., Lynch, DL., Pang, YT., Mou, Z., ... Gumbart, JC. (2021). Machine Learning Reveals the Critical Interactions for SARS-CoV-2 Spike Protein Binding to ACE2. J. Phys. Chem. Lett., 12, 5494-502.

26. Ali, A., Vijayan, R. (2020). Dynamics of the ACE2-SARS-CoV-2/SARS-CoV spike protein interface reveal unique mechanisms. Sci. Rep., 10, 14214.

27. Barros, EP., Casalino, L., Gaieb, Z., Dommer, AC., Wang, Y., Fallon, L., ... Amaro, RE. (2021). The flexibility of ACE2 in the context of SARS-CoV-2 infection. Biophys. J., 120, 1072-84.

28. Mehdipour, AR., Hummer, G. (2021). Dual nature of human ACE2 glycosylation in binding to SARS-CoV-2 spike. Proc. Natl. Acad. Sci. USA, 118

29. Williams, JK., Wang, B., Sam, A., Hoop, CL., Case, DA., Baum, J. (2021). Molecular Dynamics Analysis of a Flexible Loop at the Binding Interface of the SARSCoV-2 Spike Protein Receptor-Binding Domain. Proteins,

30. Peng, C., Zhu, Z., Shi, Y., Wang, X., Mu, K., Yang, Y., ... zhu, weiliang. (2020). Exploring the Binding Mechanism and Accessible Angle of SARS-CoV-2 Spike and ACE2 by Molecular Dynamics Simulation and Free Energy Calculation.

31. Gómez, SA., Rojas-Valencia, N., Gómez, S., Egidi, F., Cappelli, C., Restrepo, A. (2021). Binding of SARS-CoV-2 to Cell Receptors: A Tale of Molecular Evolution. Chembiochem, 22, 724-32.

32. Luan, B., Wang, H., Huynh, T. (2021). Molecular Mechanism of the N501Y Mutation for Enhanced Binding between SARS-CoV-2's Spike Protein and Human ACE2 Receptor. BioRxiv,

33. Oliveira, ASF., Ibarra, AA., Bermudez, I., Casalino, L., Gaieb, Z., Shoemark, DK., ... Mulholland, AJ. (2021). A potential interaction between the SARS-CoV-2 spike protein and nicotinic acetylcholine receptors. Biophys. J., 120, 983-93.

34. Zuckerman, DM., Chong, LT. (2017). Weighted ensemble simulation: review of methodology, applications, and software. Annu. Rev. Biophys., 46, 43-57.

35. Sztain, T., Ahn, S-H., Bogetti, AT., Casalino, L., Goldsmith, JA., Seitz, E., ... Amaro, RE. (2021). A glycan gate controls opening of the SARS-CoV-2 spike protein. Nat. Chem., 13, 963-8.

36. Casalino, L., Dommer, A., Gaieb, Z., Barros, EP., Sztain, T., Ahn, S-H., ... Amaro, RE. (2020). AI-Driven Multiscale Simulations Illuminate Mechanisms of SARS-CoV-2 
Spike Dynamics. BioRxiv,

37. Yao, H., Song, Y., Chen, Y., Wu, N., Xu, J., Sun, C., ... Li, S. (2020). Molecular Architecture of the SARS-CoV-2 Virus. Cell, 183, 730-738.e13.

38. Gur, M., Taka, E., Yilmaz, SZ., Kilinc, C., Aktas, U., Golcuk, M. (2020). Conformational transition of SARS-CoV-2 spike glycoprotein between its closed and open states. J. Chem. Phys., 153, 075101.

39. Mori, T., Jung, J., Kobayashi, C., Dokainish, HM., Re, S., Sugita, Y. (2021). Elucidation of interactions regulating conformational stability and dynamics of SARSCoV-2 S-protein. Biophys. J., 120, 1060-71.

40. Casalino, L., Gaieb, Z., Goldsmith, JA., Hjorth, CK., Dommer, AC., Harbison, AM., ... Amaro, RE. (2020). Beyond Shielding: The Roles of Glycans in the SARSCoV-2 Spike Protein. ACS Cent. Sci., 6, 1722-34.

41. Zimmerman, MI., Porter, JR., Ward, MD., Singh, S., Vithani, N., Meller, A., ... Bowman, GR. (2021). SARS-CoV-2 simulations go exascale to predict dramatic spike opening and cryptic pockets across the proteome. Nat. Chem., 13, 651-9.

42. Sikora, M., von Bülow, S., Blanc, FEC., Gecht, M., Covino, R., Hummer, G. (2021). Computational epitope map of SARS-CoV-2 spike protein. PLoS Comput. Biol., 17, e1008790.

43. Serapian, SA., Marchetti, F., Triveri, A., Morra, G., Meli, M., Moroni, E., ... Colombo, G. (2020). The Answer Lies in the Energy: How Simple Atomistic Molecular Dynamics Simulations May Hold the Key to Epitope Prediction on the Fully Glycosylated SARS-CoV-2 Spike Protein. J. Phys. Chem. Lett., 11, 8084-93.

44. Jokinen, EM., Gopinath, K., Kurkinen, ST., Pentikainen, OT. (2021). Detection of Binding Sites on SARS-CoV-2 Spike Protein Receptor-Binding Domain by Molecular Dynamics Simulations in Mixed Solvents. IEEE/ACM Trans Comput Biol Bioinform, 18, 1281-9.

45. Janik, E., Niemcewicz, M., Podogrocki, M., Saluk-Bijak, J., Bijak, M. (2021). Existing Drugs Considered as Promising in COVID-19 Therapy. Int. J. Mol. Sci., 22

46. Fantini, J., Chahinian, H., Yahi, N. (2020). Synergistic antiviral effect of hydroxychloroquine and azithromycin in combination against SARS-CoV-2: What molecular dynamics studies of virus-host interactions reveal. Int. J. Antimicrob. Agents, $56,106020$.

47. Kirby, T. (2021). New variant of SARS-CoV-2 in UK causes surge of COVID-19. Lancet Respir. Med., 9, e20-1.

48. Ramasamy, MN., Minassian, AM., Ewer, KJ., Flaxman, AL., Folegatti, PM., Owens, DR., ... Oxford COVID Vaccine Trial Group. (2021). Safety and immunogenicity of ChAdOx1 $\mathrm{nCoV}-19$ vaccine administered in a prime-boost regimen in young and old adults (COV002): a single-blind, randomised, controlled, phase 2/3 trial. Lancet, 396, 1979-93.

49. Polack, FP., Thomas, SJ., Kitchin, N., Absalon, J., Gurtman, A., Lockhart, S., ... C4591001 Clinical Trial Group. (2020). Safety and Efficacy of the BNT162b2 mRNA Covid-19 Vaccine. N. Engl. J. Med., 383, 2603-15.

50. Chung, JY., Thone, MN., Kwon, YJ. (2021). COVID-19 vaccines: The status and perspectives in delivery points of view. Adv. Drug Deliv. Rev., 170, 1-25.

51. Doshi, P., Godlee, F., Abbasi, K. (2022). Covid-19 vaccines and treatments: we must have raw data, now. BMJ, 376, o102.

52. Beigel, JH., Tomashek, KM., Dodd, LE., Mehta, AK., Zingman, BS., Kalil, AC., ... ACTT-1 Study Group Members. (2020). Remdesivir for the Treatment of Covid-19 Final Report. N. Engl. J. Med., 383, 1813-26.

53. Wang, Y., Zhang, D., Du, G., Du, R., Zhao, J., Jin, Y., ... Wang, C. (2020). 
Remdesivir in adults with severe COVID-19: a randomised, double-blind, placebocontrolled, multicentre trial. Lancet, 395, 1569-78.

54. Cohen, J. (2020). The 'very, very bad look' of remdesivir, the first FDA-approved COVID-19 drug. Science,

55. Pushpakom, S., Iorio, F., Eyers, PA., Escott, KJ., Hopper, S., Wells, A., ... Pirmohamed, M. (2019). Drug repurposing: progress, challenges and recommendations. Nat. Rev. Drug Discov., 18, 41-58.

56. Durdagi, S., Aksoydan, B., Dogan, B., Sahin, K., Shahraki, A., Birgül-İyison, N. (2020). Screening of Clinically Approved and Investigation Drugs as Potential Inhibitors of SARS-CoV-2 Main Protease and Spike Receptor-Binding Domain Bound with ACE2 COVID19 Target Proteins: A Virtual Drug Repurposing Study.

57. Durdagi, S., Orhan, MD., Aksoydan, B., Calis, S., Dogan, B., Sahin, K., ... Avsar, T. (2021). Screening of Clinically Approved and Investigation Drugs as Potential Inhibitors of SARS-CoV-2: A Combined in silico and in vitro Study. Mol. Inform., , e2100062.

58. Trezza, A., Iovinelli, D., Prischi, F., Santucci, A., Spiga, O. (2020). An integrated drug repurposing strategy for the rapid identification of potential SARS-CoV-2 viral inhibitors.

59. Day, CJ., Bailly, B., Guillon, P., Dirr, L., Jen, FE-C., Spillings, BL., ... Jennings, MP. (2021). Multidisciplinary Approaches Identify Compounds that Bind to Human ACE2 or SARS-CoV-2 Spike Protein as Candidates to Block SARS-CoV-2-ACE2 Receptor Interactions. MBio, 12

60. Lo, HS., Hui, KPY., Lai, H-M., He, X., Khan, KS., Kaur, S., ... Ng, W-L. (2021). Simeprevir Potently Suppresses SARS-CoV-2 Replication and Synergizes with Remdesivir. ACS Cent. Sci., 7, 792-802.

61. Choudhary, S., Malik, YS., Tomar, S. (2020). Identification of SARS-CoV-2 Cell Entry Inhibitors by Drug Repurposing Using in silico Structure-Based Virtual Screening Approach. Front. Immunol., 11, 1664.

62. Zhang, J., Zhou, L., Yang, Y., Peng, W., Wang, W., Chen, X. (2020). Therapeutic and triage strategies for 2019 novel coronavirus disease in fever clinics. Lancet Respir. Med., 8, e11-2.

63. Vankadari, N. (2020). Arbidol: A potential antiviral drug for the treatment of SARSCoV-2 by blocking trimerization of the spike glycoprotein. Int. J. Antimicrob. Agents, 56, 105998.

64. Razizadeh, M., Nikfar, M., Liu, Y. (2021). Small molecule therapeutics to destabilize the ACE2-RBD complex: A molecular dynamics study. Biophys. J., 120, 2793-804.

65. Coleman, CM., Sisk, JM., Mingo, RM., Nelson, EA., White, JM., Frieman, MB. (2016). Abelson kinase inhibitors are potent inhibitors of severe acute respiratory syndrome coronavirus and middle east respiratory syndrome coronavirus fusion. J. Virol., 90, 8924-33.

66. Sisk, JM., Frieman, MB., Machamer, CE. (2018). Coronavirus S protein-induced fusion is blocked prior to hemifusion by Abl kinase inhibitors. J. Gen. Virol., 99, 61930.

67. Cagno, V., Magliocco, G., Tapparel, C., Daali, Y. (2021). The tyrosine kinase inhibitor nilotinib inhibits SARS-CoV-2 in vitro. Basic Clin Pharmacol Toxicol, 128, $621-4$.

68. Hoffmann, M., Schroeder, S., Kleine-Weber, H., Müller, MA., Drosten, C., Pöhlmann, S. (2020). Nafamostat Mesylate Blocks Activation of SARS-CoV-2: New Treatment Option for COVID-19. Antimicrob. Agents Chemother., 64

69. Zhu, H., Du, W., Song, M., Liu, Q., Herrmann, A., Huang, Q. (2021). Spontaneous 
binding of potential COVID-19 drugs (Camostat and Nafamostat) to human serine protease TMPRSS2. Comput Struct Biotechnol J, 19, 467-76.

70. Nakajima, D., Negoro, N., Nakaboh, A., Nakakoji, T., Hoshiga, M., Nariyama, J., ... Hanafusa, T. (2006). Effectiveness of low dose denopamine, a beta1-adrenoceptor agonist, in a patient with vasospastic angina refractory to intensive medical treatment. Int. J. Cardiol., 108, 281-3.

71. Saint-Criq, V., Gray, MA. (2017). Role of CFTR in epithelial physiology. Cell Mol. Life Sci., 74, 93-115.

72. Raboisson, P., de Kock, H., Rosenquist, A., Nilsson, M., Salvador-Oden, L., Lin, TI., ... Simmen, K. (2008). Structure-activity relationship study on a novel series of cyclopentane-containing macrocyclic inhibitors of the hepatitis $\mathrm{C}$ virus NS3/4A protease leading to the discovery of TMC435350. Bioorg. Med. Chem. Lett., 18, 48538.

73. Sabbatini, P., Korenchuk, S., Rowand, JL., Groy, A., Liu, Q., Leperi, D., .. Kumar, R. (2009). GSK1838705A inhibits the insulin-like growth factor-1 receptor and anaplastic lymphoma kinase and shows antitumor activity in experimental models of human cancers. Mol. Cancer Ther., 8, 2811-20.

74. Chung, SSW., Cuellar, RAD., Wang, X., Reczek, PR., Georg, GI., Wolgemuth, DJ. (2013). Pharmacological activity of retinoic acid receptor alpha-selective antagonists in vitro and in vivo. ACS Med. Chem. Lett., 4, 446-50.

75. Hsu, K-L., Tsuboi, K., Chang, JW., Whitby, LR., Speers, AE., Pugh, H., Cravatt, BF. (2013). Discovery and optimization of piperidyl-1,2,3-triazole ureas as potent, selective, and in vivo-active inhibitors of $\alpha / \beta$-hydrolase domain containing 6 (ABHD6). J. Med. Chem., 56, 8270-9.

76. Mu, X-Y., Wang, R-J., Yao, Z-X., Zheng, Z., Jiang, J-T., Tan, M-Y., ... Liu, Z-H. (2019). RS 504393 inhibits M-MDSCs recruiting in immune microenvironment of bladder cancer after gemcitabine treatment. Mol. Immunol., 109, 140-8.

77. Kantarjian, H., Giles, F., Wunderle, L., Bhalla, K., O’Brien, S., Wassmann, B., ... Ottmann, OG. (2006). Nilotinib in imatinib-resistant CML and Philadelphia chromosome-positive ALL. N. Engl. J. Med., 354, 2542-51.

78. Excelra | Covid-19-Drug-Repurposing-Database.

79. Brimacombe, KR., Zhao, T., Eastman, RT., Hu, X., Wang, K., Backus, M., ... Shen, M. (2020). An OpenData portal to share COVID-19 drug repurposing data in real time. BioRxiv,

80. Galindez, G., Matschinske, J., Rose, TD., Sadegh, S., Salgado-Albarrán, M., Späth, J., ... Pauling, JK. (2021). Lessons from the COVID-19 pandemic for advancing computational drug repurposing strategies. Nat. Comput. Sci., 1, 33-41.

81. Wang, E., Sun, H., Wang, J., Wang, Z., Liu, H., Zhang, JZH., Hou, T. (2019). EndPoint Binding Free Energy Calculation with MM/PBSA and MM/GBSA: Strategies and Applications in Drug Design. Chem. Rev., 119, 9478-508.

82. Genheden, S., Ryde, U. (2015). The MM/PBSA and MM/GBSA methods to estimate ligand-binding affinities. Expert Opin. Drug Discov., 10, 449-61.

83. Barducci, A., Bonomi, M., Parrinello, M. (2011). Metadynamics. WIREs Comput Mol Sci, 1, 826-43.

84. Sabbadin, D., Moro, S. (2014). Supervised molecular dynamics (SuMD) as a helpful tool to depict GPCR-ligand recognition pathway in a nanosecond time scale. J. Chem. Inf. Model., 54, 372-6.

85. Cuzzolin, A., Sturlese, M., Deganutti, G., Salmaso, V., Sabbadin, D., Ciancetta, A., Moro, S. (2016). Deciphering the Complexity of Ligand-Protein Recognition Pathways Using Supervised Molecular Dynamics (SuMD) Simulations. J. Chem. Inf. Model., 56, 
687-705.

86. Homans, SW., Dwek, RA., Boyd, J., Mahmoudian, M., Richards, WG., Rademacher, TW. (1986). Conformational transitions in N-linked oligosaccharides. Biochemistry, 25, 6342-50.

87. Haji-Ghassemi, O., Blackler, RJ., Martin Young, N., Evans, SV. (2015). Antibody recognition of carbohydrate epitopes $\dagger$. Glycobiology, 25, 920-52. 